\title{
Implementation of Band-to-Band Tunneling Phenomena in a Multi-Subband Ensemble Monte Carlo Simulator: Application to Silicon TFETs
}

\author{
C. Medina-Bailón, J.L. Padilla, C. Sampedro, C. Alper, Student Member, IEEE, F. Gámiz, Senior Member, IEEE, \\ and A.M. Ionescu, Senior Member, IEEE.
}

\begin{abstract}
TFETs are in the way to become an alternative to conventional MOSFETs due to the possibility of achieving low subthreshold swing (SS) combined with small OFF current levels which allows operation at low $V_{\mathrm{DD}}$. In this work, a non-local band-to-band tunneling (BTBT) model has been successfully implemented into a Multi-Subband Ensemble Monte Carlo (MSEMC) simulator and applied to ultra-scaled silicon-based n-type TFETs. We have considered two different criteria for the choice of the tunneling path followed by the carriers when crossing the potential barrier, which leads to different distributions of the generated electron-hole pairs. Subband discretization due to field-induced quantum confinement has been taken into account. TCAD simulations accounting for quantization effects are considered for comparison purposes providing very accurate agreement with MS-EMC results.
\end{abstract}

Index Terms-tunnel field-effect transistors, TFET, quantum confinement, band-to-band tunneling, BTBT, Multi-Subband Ensemble Monte Carlo, MS-EMC.

\section{INTRODUCTION}

$\mathbf{I}$ $\mathrm{N}$ recent years, the basic architecture of the conventional MOSFET has been diversified to improve the device performance when transistors approach to the ultimate scaling limits. At the level of simulation research, two main working areas may be differentiated in the study of possible alternatives concerning processes and devices. The first one is mainly focused on novel engineering solutions in order to create enhanced device architectures. Its development has been conceived to improve the carrier transport properties and to keep under control the short channel effects [1], [2]. The second approach explores new device paradigms based on different injection mechanisms and the nanometric regime enforced by scaling.

Among some of the most promising solutions in the latter area, this work deals with one of the devices proposed to reach sub-60 $\mathrm{mV} / \mathrm{dec}$ subthreshold swings $(\mathrm{SS})$ : the tunnel fieldeffect transistor (TFET) [3]. In practice, the best MOSFET implementations cannot bring SS below $70-80 \mathrm{mV} / \mathrm{dec}$, which

This work was supported by the European Community's Seventh Framework Programme Marie Curie Action under Grant Agreement No. 291780 (Programme Andalucía Talent Hub); Horizon 2020 projects REMINDER (Grant No. 687931), WAYTOGO-FAST (Grant No. 662175), and the Spanish Ministry of Economy under grant agreement TEC2014-59730-R.

C. Medina-Bailón, J.L. Padilla, C. Sampedro and F. Gámiz are with the Departamento de Electrónica y Tecnología de los Computadores. Universidad de Granada. Avda. Fuentenueva s/n, 18071 Granada, Spain (e-mail: cmedba@ugr.es).

C. Alper and A.M. Ionescu are with the Nanoelectronic Devices Laboratory, École Polytechnique Fédérale de Lausanne, Lausanne CH-1015, Switzerland. leads to even worse situations [4]-[6] with the subsequent limitation in the reduction of $V_{\mathrm{DD}}$. On the other hand, TFETs should not be constrained by the aforementioned $60 \mathrm{mV} / \mathrm{dec}$ limit and, therefore, arise as potential substitutes for conventional MOSFET in low-power applications. The process of quantum mechanical tunneling through a barrier between energy bands, known as band-to-band tunneling (BTBT), governs the injection of carriers in TFETs, contrary to MOSFETs where thermionic emission dominates.

In this work, we present a comparison in the study of ultrascaled silicon-based n-type TFETs using two different simulation approaches. The first one is a Multi-Subband Ensemble Monte Carlo (MS-EMC) simulator specifically designed for this aim. The second (used for comparison purposes) is a customized TCAD-based approach including quantum effects that has extensively been used in the last years [7], [8]. Furthermore, for the MS-EMC, we assess the impact on the BTBT generation rate distribution of two different tunneling path choices. The first one estimates dinamically the BTBT path according to the criterion of the valence band maximum gradient trajectory; and the second one searches for the path featuring the minimum length trajectory.

The paper is organized as follows: Section II introduces the analyzed device, outlines the methodology to account for field-induced subband discretization, provides a detailed overview of the BTBT model used in the MS-EMC code and describes the TCAD-based simulation approach. Simulation results and discussion are presented in Section III. Finally, the main conclusions are drawn in Section IV.

\section{Device Structure And Methodology}

\section{A. Device Structure}

The simulated n-type TFET is schematically depicted in Fig. 1 along with its doping concentrations and dimensions. The center of the device in the $x$ direction corresponds to $x=0 \mathrm{~nm}$. It features an $\mathrm{n}$-doped drain with $N_{\mathrm{D}}=10^{19} \mathrm{~cm}^{-3}$, a p-type doped source with $N_{\mathrm{S}}=10^{20} \mathrm{~cm}^{-3}$, and an n-doped channel with $10^{15} \mathrm{~cm}^{-3}$, a gate oxide with EOT $=1 \mathrm{~nm}$, gate workfunctions of $4.05 \mathrm{eV}$, and a body thickness ranging from $T_{\mathrm{Si}}=5 \mathrm{~nm}$ to $T_{\mathrm{Si}}=7 \mathrm{~nm}$.

\section{B. Subband Quantization Corrections}

Both simulation techniques contained in this work, the MSEMC simulator and the customized TCAD approach, implement non-local direct and phonon assisted BTBT taking into 


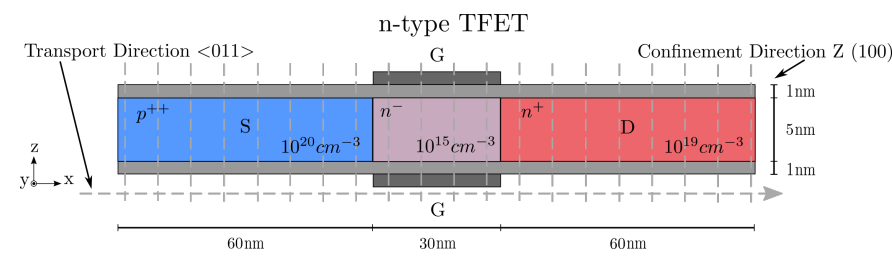

Figure 1: Si n-type TFET analyzed in this work. 1D Schrödinger equation is solved for each grid point in the transport direction and BTE is solved by the MC method in the transport plane.

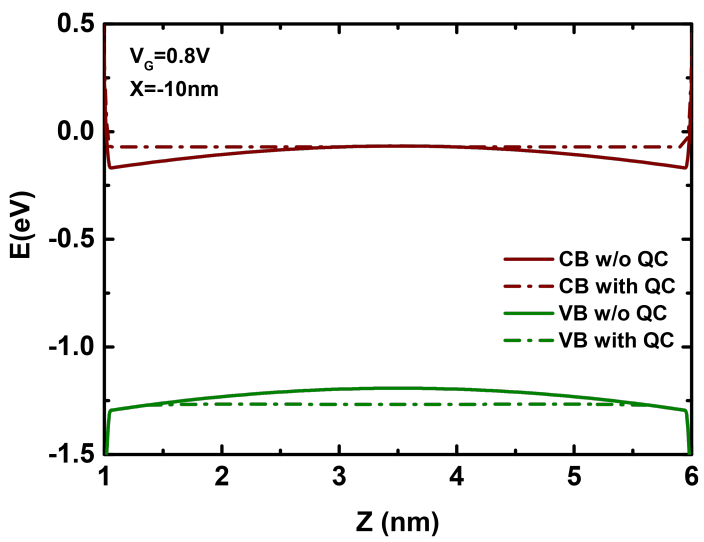

Figure 2: Quantum corrections included in the MS-EMC for the conduction and valence bands at $V_{\mathrm{GS}}=0.8 \mathrm{~V}, V_{\mathrm{DS}}=1 \mathrm{~V}$ and $x=-10 \mathrm{~nm}$. Recall that $x=0 \mathrm{~nm}$ corresponds to the center of the device.

account quantum confinement effects through discretization of conduction and valence bands into energy subbands. Should this correction be neglected, carriers would continue being injected at/from the edges of the bands with the subsequent error that it implies given that those energy levels now become forbidden states. So as to guarantee that BTBT takes place between first bound states, both simulators need to include a band profile modification algorithm allowing the reshaped conduction and valence bands to match their first subbands, $E_{e 1}$ and $E_{h 1}$, respectively. Fig. 2 illustrates a particular example of band modification obtained from the MS-EMC for a vertical cut taken at $x=-10 \mathrm{~nm}$. The specific implementation of quantum corrections in each of the simulators is outlined in the following sections along with their detailed description.

\section{BTBT Setup in MS-EMC}

The BTBT model included in the MS-EMC code is based on the mode-space approach of quantum transport [9]. The structure of the device is divided into slices along the confinement direction where the 1D Schrödinger equation is solved, whereas the 2D Bolzmann Transport Equation (BTE) is solved in the transport plane for electrons (XY in Fig. 1). The selfconsistency of the solution for these carriers is preserved by coupling these two equations to the 2D Poisson equation. However, since holes travel mostly in the low field source region, a drift-diffusion approach is used to describe them. This MS-EMC tool has already demonstrated its capabilities in different scenarios where the performance of advanced nanodevices has been studied [10], [11]. In spite of the scattering mechanisms and the quantum effects included in this code, its reasonable computational effort is one of the main advantages of this approach with respect to the fullquantum approach. In addition, the quantum transport effects can be included in a separate way thanks to the decoupled approximation [12]. Taking advantage of this, the BTBT model has been incorporated in additional blocks modifying the MSEMC simulator as illustrated in the flowchart represented in Fig. 3. This BTBT treatment accounts for the tunneling current contribution through suitable generation rate functions, $G_{\mathrm{BTBT}}$, for electrons and holes in the conduction and valence bands, respectively [13]. This approximation simplifies the treatment of the BTBT phenomena and allows low computational cost.

If we now focus on the BTBT block of Fig. 3, the first box corresponds to the aforementioned subband quantization corrections for the conduction and valence bands. This scheme of operation is necessary because the carriers are initially placed into subbands and, at a given $x$, their position distribution in $z$ is set by the solution of the Schrödinger equation. Therefore, an adequate mapping procedure between $2 \mathrm{D}$ conduction and valence bands and their respective subbands is required.

Since the 1D Schrödinger equation is solved for electrons, their first subband, $E_{e 1}$, is known. Then, at every vertical slice (fixed $x$ ), for those points in the conduction band verifying $E_{\mathrm{C}}(z) \leq E_{e 1}$, we set $E_{\mathrm{C}}(z)=E_{e 1}$. As for the hole treatment, the valence band profile can be accurately approximated along $z$ by a parabolic well [14] as inferred from Fig. 2. Therefore, for fixed $x$, the modified valence band would read as

$$
E_{\mathrm{V}, \text { modif }}(z)=E_{\mathrm{V}}(z)-\Delta E_{h 1}(z),
$$

where $\Delta E_{h 1}(z)$ is a position dependent quantity that makes use of the analytical resolution of the parabolic well profile as

$$
\Delta E_{h 1}(z)=\left\{\begin{array}{ll}
\hbar \sqrt{\frac{|k(z)| q}{m_{h}^{*}}}-\frac{1}{2}|k(z)| d^{2} & \text { if } \Delta E_{h 1}(z)>0 \\
0 & \text { if } \Delta E_{h 1}(z) \leq 0
\end{array},\right.
$$

with $q$ the electron charge, $k(z)=V^{\prime \prime}(z)$ and $m_{h}^{*}$ the hole effective mass. For each vertical slice, $d$ is the distance from the center of the parabola. Notice how this treatment for holes is more realistic than simply considering for them a rectangular well approximation [13].

Once the bands have been appropriately corrected to match their first subbands, the next step is the determination of the starting and ending points for the tunneling processes. These points are estimated by calculating the path followed by the carriers when they tunnel across the forbidden energy barrier. One on the main advantages of our MS-EMC code is that it allows dynamical determination of the $2 \mathrm{D}$ tunneling path according to the up-to-date electrostatic configuration at each simulation step.

Two different assumptions have been considered here [15] for calculating the tunneling path in the corresponding box of Fig. 3. The first one estimates the path following the criterion of the valence band "maximum gradient trajectory" (i.e. maximum electric field, $F_{\max }$ ). In this case, the tunneling 


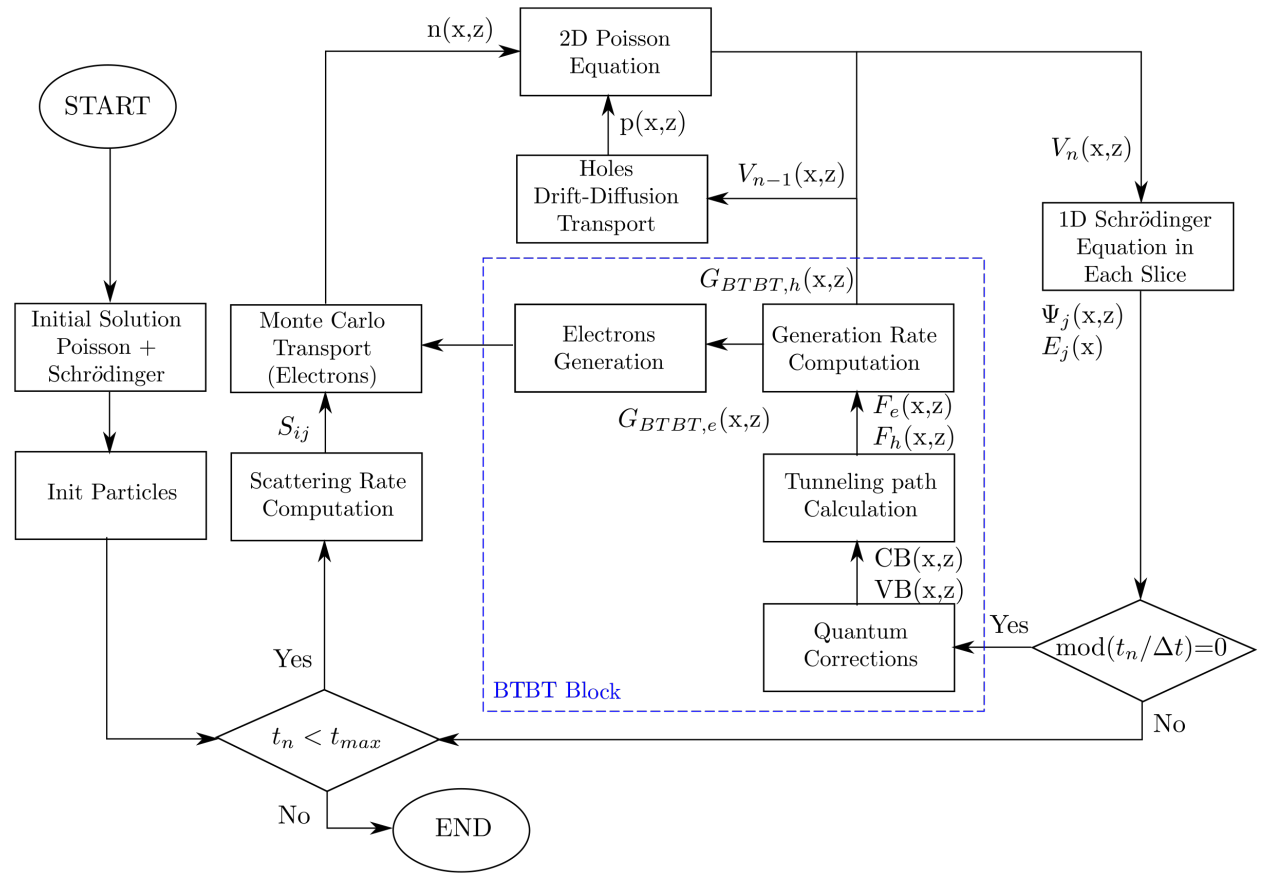

Figure 3: Flowchart of the MS-EMC simulator with the additional blocks of the BTBT code where $x$ is the transport direction, $z$ the confinement direction, $n(x, z)$ and $p(x, z)$ are the electron and hole concentrations, respectively, $V(x, z)$ is the potential profile, $E_{j}(x)$ is the subband energy, $\Psi_{j}(x, z)$ are the subband eigenfunctions, $S_{i j}$ are the scattering rates, subscript $n$ stands for the iteration number, $\Delta t$ is the time step where BTBT is calculated, $F_{e}(x, z)$ and $F_{h}(x, z)$ are the electric fields of electrons and holes associated to the selected tunneling path, $G_{\mathrm{BTBT}, e}(x, z)$ and $G_{\mathrm{BTBT}, h}(x, z)$ are the electron and hole generation rates, respectively.

path is dynamically computed based on the self-consistent potential computed during the simulation. It is then easy to understand that for the $F_{\max }$ method, as the calculated paths

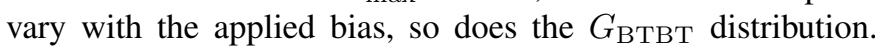
The second way of determining the tunneling path follows the "minimum length" $\left(L_{\min }\right)$ criterion. If we had to privilege one of the two assumptions, we would be more tempted to tend toward the $F_{\max }$ method given that, as the tunneling region is a zone of high electric field, the valence band electrons allowed to tunnel would be more likely expected to do it in a direction equal to the force that is pushing them towards the potential barrier. For that reason, this tunneling path would be in principle more probable from a purely physical point of view. Nonetheless, as the tunneling process is not an intuitive classical event, it should not be regarded as such. Therefore, if one considers that carriers could potentially tunnel in many directions, it could be also reasonable to assume that, for example, electrons choose a tunneling path in which they jump from the valence band to the nearest equi-energetic point in the conduction band (i.e. $L_{\min }$ method).

The choice of the non-local tunneling path is very relevant in the calculation of the BTBT rates because we need it to identify the starting and ending points of the tunneling process to estimate an "a posteriori" local effective electric field (whose determination only requires those two points and the distance between them), which, in turn, is used to mimic the actual non-local scenario through the utilization of local equations (later shown). The non-locality is therefore greatly captured by this method since, by determining our tunneling path, we are dynamically accounting for the self-consistent

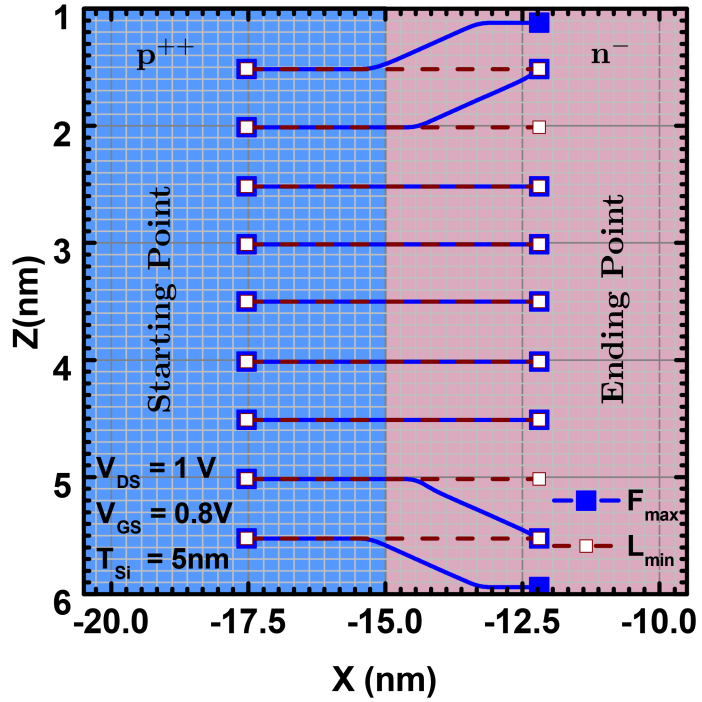

Figure 4: Some examples of different tunneling paths estimated using the two criteria considered in our work $\left(F_{\max }\right.$ and $\left.L_{\min }\right)$. Recall that $x=-15 \mathrm{~nm}$ corresponds to the limit between the source and the channel.

modification of the conduction and valence bands throughout the successive Monte Carlo iterations. As an example, Fig. 4 shows some examples of different tunneling paths estimated using the two criteria considered in our work.

Still in the box corresponding to the tunneling path calculation, notice that two types of electric fields will need to be considered. One of them for electrons, $F_{e}(x, z)$, and 
the other one for holes, $F_{h}(x, z)$. It is important to highlight the difference between them, as their corresponding carriers effectively follow independent paths. In other words, two generated electrons can reach the same ending point in the conduction band, whereas their counterpart holes could have been generated at different starting points in the valence band. As a result of this, the tunneling generation rates are entirely dependent on: $i$ ) the local electric field at each point, $i i$ ) on the full tunneling barrier profile, and iii) on the selected tunneling path.

The next step is to obtain $G_{\mathrm{BTBT}}$ for electrons and holes. Our calculation is based on the Kane's model applied to the BTBT generation rate per unit of volume [16]-[18]:

$$
\begin{aligned}
& G_{\mathrm{BTBt}, e}(x, z)=A\left(\frac{F_{e}(x, z)}{F_{0}}\right)^{P} \exp \left(-\frac{B}{F_{e}(x, z)},\right) \\
& G_{\mathrm{BTBT}, h}(x, z)=A\left(\frac{F_{h}(x, z)}{F_{0}}\right)^{P} \exp \left(-\frac{B}{F_{h}(x, z)},\right)
\end{aligned}
$$

where $F_{0}=1 \mathrm{~V} / \mathrm{m}, \mathrm{P}=2.5$ for the phonon assisted tunneling process. The prefactor $\mathrm{A}$ and the exponential factor $\mathrm{B}$ for indirect transitions read as [19]

$$
\begin{gathered}
A=\frac{g\left(m_{v} m_{c}\right)^{3 / 2}\left(1+2 N_{T A}\right) D_{T A}^{2}\left(q F_{0}\right)^{5 / 2}}{2^{21 / 4} h^{5 / 2} m_{r}^{5 / 4} \rho \epsilon_{T A}\left[E_{g}(300 K)+\Delta_{c}\right]^{7 / 4}}, \\
B=\frac{2^{7 / 2} \pi m_{r}^{1 / 2}\left[E_{g}(300 K)+\Delta_{c}\right]^{3 / 2}}{3 q h},
\end{gathered}
$$

where $\mathrm{g}$ is a degeneracy factor, $m_{c}$ and $m_{v}$ are the conduction and valence band density of states effective masses, respectively, $m_{r}$ is the reduced tunneling mass, $N_{T A}$ is the occupation number of the transverse acoustic phonons at temperature T, $D_{T A}$ is the deformation potential of transverse acoustic phonons, and $\epsilon_{T A}$ is the transverse acoustic phonon energy. The rest of the parameters takes their usual meaning. In this approach, only the transverse acoustic phonons are taken into account because they have the highest phonon occupation number and the smallest phonon energy [20].

Once the tunneling paths and $G_{\mathrm{BTBT}}$ are calculated, the last box inside the BTBT block of Fig. 3 translates them into generated charges in the MS-EMC in a self-consistent way [13]. As mentioned above, a drift diffusion approach is considered to describe the hole transport as they are mainly generated in the source. They are not treated as individual particles and thus a correction in the hole concentration is simply added to account for the generated holes at each time step $\left(\Delta p_{\mathrm{BTBT}}(x, z)=G_{\mathrm{BTBT}, h}(x, z) \cdot \Delta t\right)$ following the explicit expression for this aim extracted from [21] regarding the utilization of the Scharfetter-Gummel discretization scheme. A different methodology applies for electrons since, for them, a number of superparticles, $N_{e}$, is generated in the BTBT process. This $N_{e}$ generation depends on the time step, $\Delta t$, the statistical weight, $w$, and the electron generation rate $G_{\mathrm{BTBT}, e}(x, z)$ at the position of the superparticle. The procedure is as follows: first, these superparticles are generated in the fundamental subband with randomly chosen $x$ inside the considered grid cell with a probability distribution given by $P(x)$ due to the 2D MS-EMC approximation:

$$
P(x)=\frac{\int_{0}^{T_{\mathrm{Si}}} G_{\mathrm{BTBT}, e}(x, z) d z}{\int_{L_{x}} \int_{0}^{T_{\mathrm{Si}}} G_{\mathrm{BTBT}, e}(x, z) d x d z} .
$$

Second, the number of particles is calculated taking into account the corresponding generation rate for the considered slice $x_{i}$ :

$$
N_{e}=\frac{\Delta t}{w} \int_{0}^{T_{\mathrm{Si}}} G_{\mathrm{BTBT}, e}\left(x_{i}, z\right) d z
$$

Note that the fact of determining in a certain way (either via $F_{\max }$ or $L_{\min }$ ) the BTBT path inside the forbidden energy barrier implies the consideration of the spatial variation of the bands across that path. This is why the methodology described in this section accounts for non-locality phenomena.

In order to optimize the computational load, the time step $\Delta t$ where BTBT is calculated can be chosen independently of the Monte Carlo time step $\left(t_{n}\right)$. The inclusion of new superparticles in the MS-EMC increases the simulation time because we need to solve the free-flight block of each one regardless of its weight $(w)$. For this reason, it is worth choosing $t_{n}$ so as to assign an adequate number of electrons per superparticle. Finally, a maximum tunneling rejection length, $L_{\max }$, is also introduced so that, if up to an given integration step the tunneling length of a particle turns out to be higher than $L_{\max }$, the calculation of its tunneling path stops. $L_{\max }$ has been chosen to match the channel length, $L_{\max }=L_{G}=30 \mathrm{~nm}$.

\section{TCAD Simulation Approach}

The TCAD simulation scheme that we follow using Synopsys [19] is similar to that previously discussed and tested in recent works [7], [22]. However, the nature of the device herein analyzed allowed us to modify it in order to speed up the simulations execution. The particularity of this device is that it features band profiles easily approximable by triangular wells (for electrons in the conduction band) and parabolic wells (for holes in the valence band) as observed in Fig. 2. Therefore, we can replace in this case the Schrödinger-Poisson resolution by an analytical estimation of the subbands with great accuracy and significant saving in simulation time.

The analytical handling for holes in this TCAD approach is exactly the same as that described by Eqs. 1 and 2 and is included in Synopsys through the so-called physical model interface (PMI) that allows to access and modify certain models of the simulator by adequate $\mathrm{C}++$ subroutines. As for electrons, an analogue technique with its corresponding subroutine can be implemented for each $x$ [23], [24].

Regarding BTBT, we account for it by means of the dynamic nonlocal BTBT model of Sentaurus [19] which dynamically calculates the tunneling paths based on the energy band profiles. The idea is that prior to injecting the carriers, 


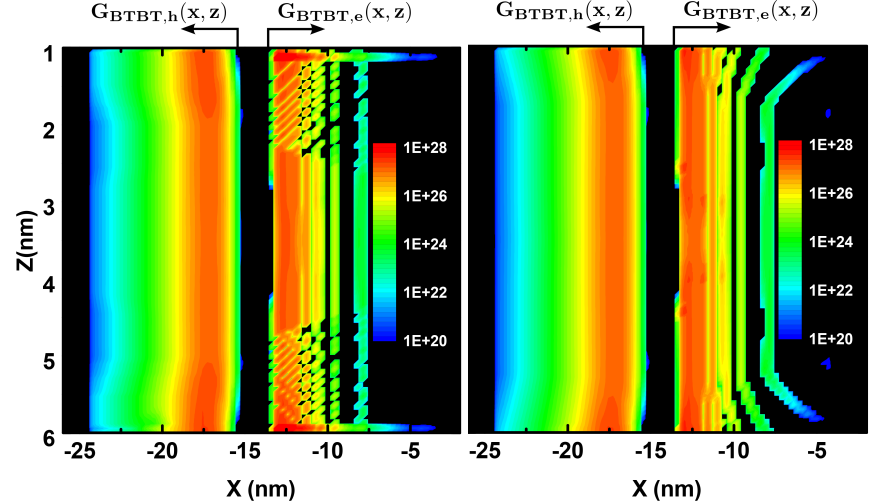

(a)

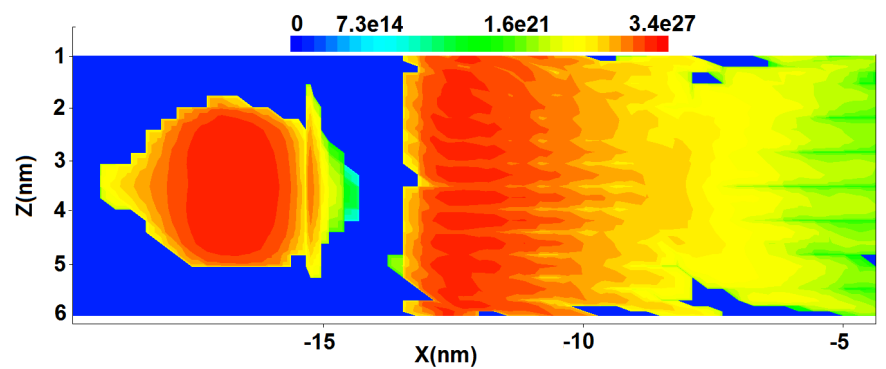

(c)

Figure 5: Generation rate distributions for holes and electrons. (a) MS-EMC with tunneling path following the maximum gradient trajectory, (b) MS-EMC with minimum length tunneling path, (c) TCAD-based approach with quantum corrections included. All figures correspond to $T_{\mathrm{Si}}=5 \mathrm{~nm}, V_{\mathrm{GS}}=0.8 \mathrm{~V}, V_{\mathrm{DS}}=1 \mathrm{~V}$. The center of the device is taken as reference position with $x=0 \mathrm{~nm}$. The color scale is in $\mathrm{cm}^{-3} \mathrm{~s}^{-1}$.

we modify the profiles of the conduction and valence bands (making use of the aforementioned subroutines) which makes them coincident with their first bound states. By doing so, we manage BTBT to occur between first subbands, and not between band edges as it happened semiclassically in the absence of quantization effects. Other TCAD-based bandgap widening techniques can also be found in the literature [25][27].

\section{Results AND Discussion}

As described in Sec. II-C, two different tunneling trajectories have been considered to mimic in a semiclassical way the quantum mechanical phenomenon of interband tunneling. Both tunneling paths (the one following the maximum gradient and the one accounting for the minimum tunneling length) are an acceptable bet for including in the MS-EMC simulator a feasible BTBT model. Accepting this premise, the different spatial distributions of the BTBT generation rates obtained from each method ( $F_{\max }$ and $L_{\text {min }}$, depicted in Fig. 5(a) and (b)) should not be interpreted as the evidence of an inconsistency in the simulation approach, but as the proof of the conceptual difference between two independent ways of modeling the mentioned semiclassical trajectories. It is worth noticing that the $L_{\min }$ criterion tends to distribute the electron generation uniformly inside the channel along the $z$-direction, whereas $F_{\max }$ provides a generation profile more concentrated towards the gate dielectrics and nearer to the source-to-channel junction. For the sake of comparison, Fig. 5(c) shows the mapping of $G_{\mathrm{BTBT}}$ obtained for the same biasing and from TCAD-based simulations.

One important and pertinent question arises in light of the different $G_{\text {BTBT }}$ profiles obtained in each case: does this difference in the generation rate distributions entail a difference in the total number of carriers injected by BTBT? The answer to this question is given in Fig. 6 where we show the total number of electrons generated in the channel by means of BTBT for the MS-EMC (considering both $F_{\max }$ and $L_{\min }$ ) and for the TCAD approach. One of the main advantages of the method implemented in our MS-EMC code is that it allows to inject in the most probable slice (from Eq. 7) the number of generated carriers corresponding to that slice (from Eq. 8), and not the total number of carriers (as done in [13]). As a result, Fig. 6 is not the BTBT rate multiplied by a certain time, but the average of the generated electrons. Observe that overall there is a good matching between the displayed curves suggesting that our BTBT treatment in the MS-EMC provides accurate results for this type of devices.

Moreover, as the total number of carriers injected by BTBT in this TFET device turns out to be mostly negligible in comparison with the total carrier distribution inside the channel, the tunneling path choice does not have a noticeable impact on the energy profile of the lower subband, $E_{e 1}$. This can be observed for $V_{\mathrm{DS}}=1 \mathrm{~V}$ and $V_{\mathrm{GS}}=0.8 \mathrm{~V}$ in Fig. 7 along with the corresponding subband profile obtained from TCAD simulations.

Finally, the transfer characteristics calculated from the integration of the BTBT generation rate along the selected (most probable) slice in the MS-EMC simulations are shown in Fig. 8. Again, the comparison with the results arising from the TCAD-based approach suggests a good performance of our MS-EMC even in the low subthreshold region. This confirms the fact that the Monte Carlo method is also suitable for assessing this type of devices at very reduced current levels, which traditionally was known to be a problematic issue. Of course, the extremely reduced $\mathrm{ON}$ currents featured by the analyzed device are due to the fact that we are dealing with silicon. Once that the MS-EMC simulator has proven to be reliable for handling BTBT phenomena, next steps would be oriented to the consideration of direct materials with lower bandgaps and alternative geometries like that of the heterogate EHBTFET or the Fin EHBTFET.

\section{CONCLUSIONS}

This work presents the implementation of a non-local BTBT model inside an existing MS-EMC tool for the study of ultrascaled TFETs allowing a detailed scattering description and a moderate computational cost. The necessary semiclassical adaptation of the quantum mechanical process of interband tunneling led us to develop two alternative methods for defining the concept of tunneling path inside the forbidden barrier. Quantum corrections associated to subband discretization phenomena have been considered for both electrons and holes. Results from TCAD simulations including quantization effects 

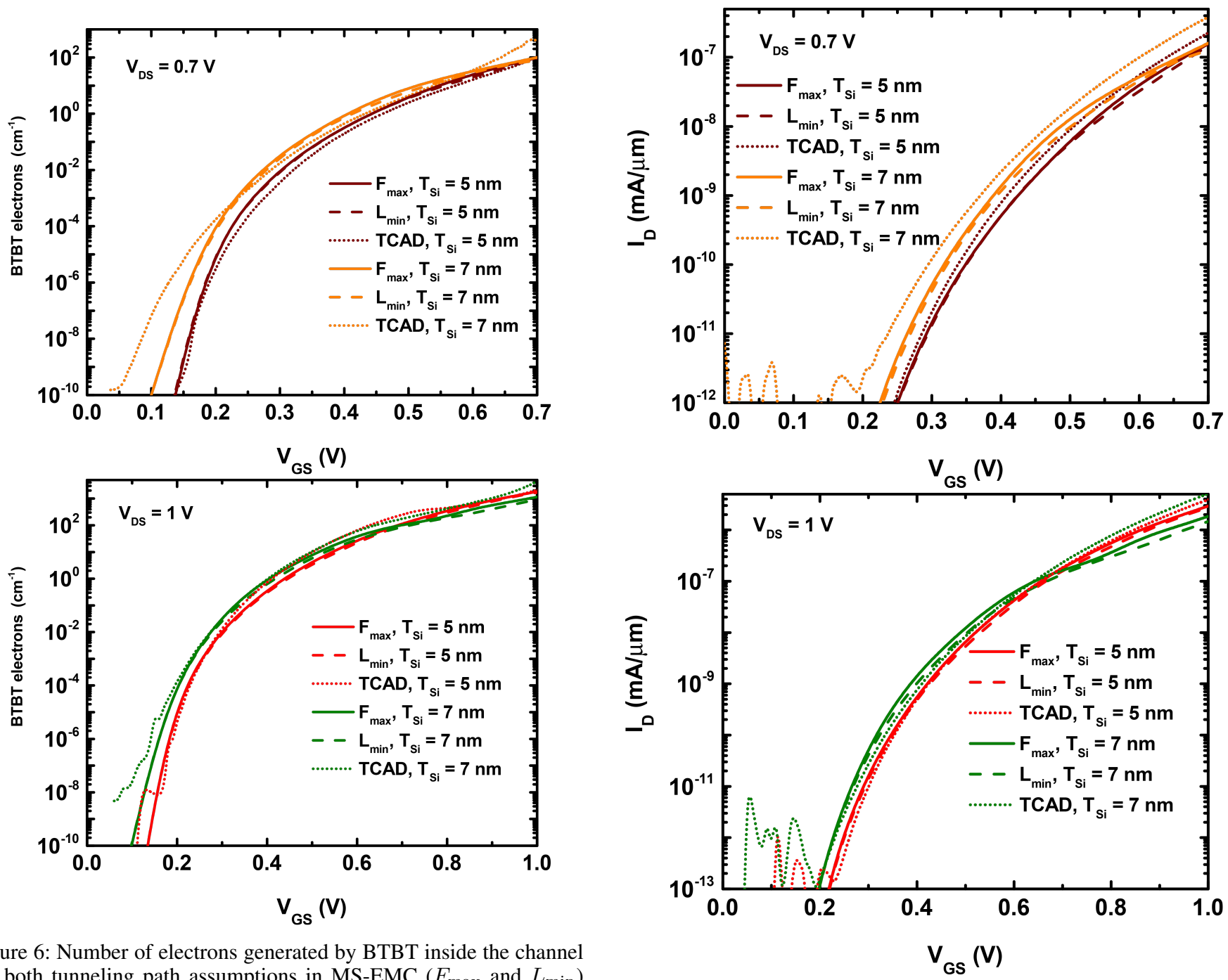

Figure 6: Number of electrons generated by BTBT inside the channel for both tunneling path assumptions in MS-EMC ( $F_{\max }$ and $\left.L_{\min }\right)$ compared to the result obtained from the TCAD-based simulation approach at $V_{\mathrm{DS}}=0.7 \mathrm{~V}$ (top) and $V_{\mathrm{DS}}=1 \mathrm{~V}$ (bottom) with $T_{\mathrm{Si}}=$ $5 \mathrm{~nm}$ and $T_{\mathrm{Si}}=7 \mathrm{~nm}$.

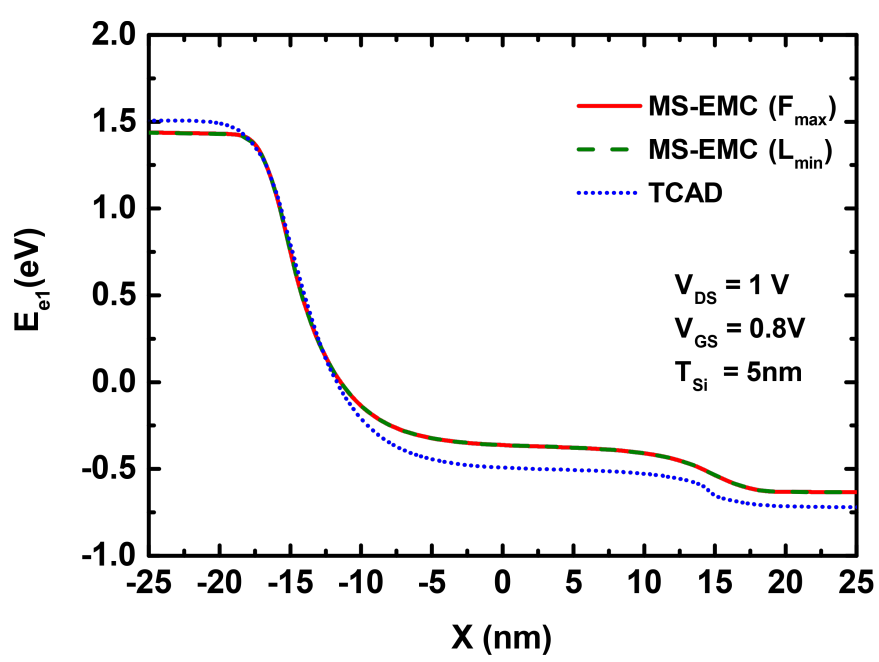

Figure 7: Behavior of the first subband for electrons following the $x$ direction. As expected, the two different tunneling path criteria inside the MS-EMC provide almost identical profiles.
Figure 8: $I_{\mathrm{D}}-V_{\mathrm{GS}}$ curves obtained with the MS-EMC (for both $F_{\max }$ and $L_{\min }$ methods) compared to the one resulting from the TCADbased approach at $V_{\mathrm{DS}}=0.7 \mathrm{~V}$ (top) and $V_{\mathrm{DS}}=1 \mathrm{~V}$ (bottom) with $T_{\mathrm{Si}}=5 \mathrm{~nm}$ and $T_{\mathrm{Si}}=7 \mathrm{~nm}$. Reduced ON current levels are due to the utilization of silicon as channel material.

have been used for comparison with the proposed simulation approach.

\section{REFERENCES}

[1] K. J. Kuhn, "Considerations for ultimate CMOS scaling," IEEE Transactions on Electron Devices, vol. 59, no. 7, pp. 1813-1828, 2012.

[2] M. Bohr, "The Evolution of Scaling from the Homogeneous Era to the Heterogeneous Era.” 2011 IEEE International Electron Devices Meeting (IEDM), 2011, pp. 1-6.

[3] A. Ionescu and H. Riel, "Tunnel field-effect transistors as energyefficient electronic switches," Nature, vol. 479, pp. 329-337, November 2011.

[4] H.-S. Wong, "Beyond the conventional transistor," IBM Journal of Research and Development, vol. 46, pp. 133-168, March 2002.

[5] J. Colinge, J. Alderman, W. Xiong, and C. Cleavelin, "Quantummechanical effects in trigate SOI MOSFETs," IEEE Transactions on Electron Devices, vol. 53, pp. 1131-1136, May 2006.

[6] C. Lee, A. Nazarov, I. Ferain, N. D. Akhavan, R. Yan, P. Razavi, R. Yu, R. Doria, and J. Colinge, "Low subthreshold slope in junctionless multigate transistors," Journal of Applied Physics, vol. 96, no. 102106, March 2010. 
[7] J. L. Padilla, C. Alper, A. Godoy, F. Gamiz, and A. M. Ionescu, "Impact of Asymmetric Configurations on the Heterogate Germanium ElectronHole Bilayer Tunnel Field-Effect Transistor Including Quantum Confinement," IEEE Transactions on Electron Devices, vol. 62, no. 11, pp. 3560-3566, 2015.

[8] J. L. Padilla, C. Alper, C. Medina-Bailon, F. Gamiz, and A. M. Ionescu, "Assessment of pseudo-bilayer structures in the heterogate germanium electron-hole bilayer tunnel field-effect transistor," Applied Physics Letters, vol. 106, no. 26, pp. $262102-1-262$ 102-4, 2015.

[9] R. Venugopal, Z. Ren, S. Datta, M. S. Lundstrom, and D. Jovanovic, "Simulating quantum transport in nanoscale transistors: Real versus mode-space approaches," Journal of Applied Physics, vol. 92, no. 7, pp. 3730-3739, 2002.

[10] C. Sampedro, F. Gámiz, A. Godoy, R. Valín, A. García-Loureiro, and F. G. Ruiz, "Multi-Subband Monte Carlo study of device orientation effects in ultra-short channel DGSOI," Solid-State Electronics, vol. 54, no. 2, pp. 131-136, 2010.

[11] C. Sampedro, F. Gámiz, and A. Godoy, "On the extension of ET-FDSOI roadmap for $22 \mathrm{~nm}$ node and beyond," Solid-State Electronics, vol. 90, pp. 23-27, 2013.

[12] C. Medina-Bailon, C. Sampedro, F. Gámiz, A. Godoy, and L. Donetti, "Confinement orientation effects in S / D tunneling," p. Available Online, 2016.

[13] A. Revelant, P. Palestri, and L. Selmi, "Multi-subband semi-classical simulation of n-type Tunnel-FETs," 2012 13th International Conference on Ultimate Integration on Silicon (ULIS), pp. 187-190, Mar. 2012.

[14] O. Manasreh, "Parabolic potential well," in Introduction to nanomaterials and devices. New Jersey: Willey, 2011, pp. $437-441$.

[15] L. De Michielis, M. Iellina, P. Palestri, A. M. Ionescu, and L. Selmi, "Effect of the choice of the tunnelling path on semi-classical numerical simulations of TFET devices," Solid-State Electronics, vol. 71, pp. 7-12, May 2012.

[16] E. O. Kane, "Zener tunneling in semiconductors," Journal of Physics and Chemistry of Solids, vol. 12, no. 2, pp. 181 - 188, 1960.

[17] — , "Theory of Tunneling," Journal of Applied Physics, vol. 32, no. 1, p. $83,1961$.

[18] W. Vandenberghe, B. Sorée, W. Magnus, and M. V. Fischetti, "Generalized phonon-assisted Zener tunneling in indirect semiconductors with non-uniform electric fields: A rigorous approach," Journal of Applied Physics, vol. 109, no. 12, p. 124503, 2011.

[19] Synopsys, "Sentaurus Device User Version 2014.09," no. September, 2014.

[20] K.-H. Kao, A. S. Verhulst, W. G. Vandenberghe, B. Soree, G. Groeseneken, and K. De Meyer, "Direct and Indirect Band-to-Band Tunneling in Germanium-Based TFETs," IEEE Transactions on Electron Devices, vol. 59, no. 2, pp. 292-301, 2012.

[21] S. Selberherr, Analysis and Simulation of Semiconductor Devices. New York: Springer-Verlag, 1984.

[22] J. L. Padilla, C. Alper, F. Gamiz, and A. M. Ionescu, "Quantum Mechanical Confinement in the Fin Electron?Hole Bilayer Tunnel FieldEffect Transistor," IEEE Transactions on Electron Devices, vol. 63, no. 8, pp. 3320-3326, 2016.

[23] S. Sant and A. Schenk, "Methods to Enhance the Performance of InGaAs/InP Heterojunction Tunnel FETs," IEEE Transactions on Electron Devices, vol. 63, no. 5, pp. 2169 - 2175, 2016.

[24] W. G. Vandenberghe, Sorée, B., W. Magnus, G. Groeseneken, A. S. Verhulst, and M. V. Fischetti, "Field induced quantum confinement in indirect semiconductors: Quantum mechanical and modified semiclassical model." In 2011 International Conference on Simulation of Semiconductor Processes and Devices (SISPAD), 2011, pp. 271-274.

[25] A. M. Walke, A. S. Verhulst, A. Vandooren, D. Verreck, E. Simoen, V. R. Rao, G. Groeseneken, N. Collaert, and A. V. Y. Thean, "Part I: Impact of Field-Induced Quantum Confinement on the Subthreshold Swing Behavior of Line TFETs," IEEE Transactions on Electron Devices, vol. 60, no. 12, pp. 4057-4064, 2013.

[26] J. L. Padilla, F. Gamiz, and A. Godoy, "A Simple Approach to Quantum Confinement in Tunneling Field-Effect Transistors," IEEE Electron Device Letters, vol. 33, no. 10, pp. 1342-1344, 2012.

[27] G. B. Beneventi, E. Gnani, A. Gnudi, S. Reggiani, and G. Baccarani, "Optimization of a Pocketed Dual-Metal-Gate TFET by Means of TCAD Simulations Accounting for Quantization-Induced Bandgap Widening," IEEE Transactions on Electron Devices, vol. 62, no. 1, pp. 44-51, 2015. 